\title{
Generation of Point Scatterer Models Using PTD/SBR Technique
}

\author{
Shuen-Yih Wang and Shyh-Kang Jeng* \\ Rm. 545, Department of Electrical Engineering, National Taiwan University \\ Taipei, Taiwan, R.O.C.
}

\section{Introduction}

In radar scattering problems, a target can be represented by a finite number of discrete point scatterers, each of which produces a scattered wave of specified amplitude and phase. This is oiten called the point scatterer mudel and these point scatterers are otten called scattering centers. This model was first used to analyze the radar glint [1] and is recently implemented in the real-time, hardware-in-the-loop (HWIL) simulation [2]. There were two ways to assign the position, the amplitude and the phase of each scattering center. The first is to assign their statistics which can be used to develop statistical models for RCS or glint [3],[4]. The second is to get the inverse synthetic aperture radar (ISAR) images from measurement or simulation data and process the images to generate the point scatterers [2]. A deterministic method which does not need the ISAR imaging will be proposed in this paper. It is believed to be useful for simulation purpose.

\section{Formulation}

In the point scatterer model, the monostatic RCS of a target can be expressed as

$$
\sigma_{u v}=\left|\sum_{n=1}^{N} \sqrt{\sigma}_{u v, n} e^{-2 \jmath k\left|\bar{r}_{0}-\tilde{r}_{n}\right|}\right| \quad u, v=\theta, \phi
$$

where $\sigma_{u v}$ is the total RCS in $u$ polarization due to an incident wave in $v$ polarization, $\sqrt{\sigma}_{u v, n}$ is the phasor representing the amplitude and phase of the $n$th scattering center, $\bar{r}_{o}$ is the location of the receiver, $\bar{r}_{n}$ is the position of the nth scattering center and $\mathrm{k}$ is the wave number. We will illustrate below that the high frequency scattering mechanism, including single reflection, single diffraction and multiple reflection, can be put into the form of Eq.(1). Assume that the target is composed of triangular facets.

The single reflection can be put into the form of Eq.(1) by physical optics (PO). Through PO, we find that the PO integral over each illuminated facet is related to $\sqrt{\sigma}_{u v, n}$ and the center of each facet is assign to be $\bar{r}_{n}$. Similarly, the single diffraction can be treated by physical theory of diffraction (P'D). It is found that the scattered field by the PT'D method for the lit edge segments is related to $\sqrt{\sigma}_{u v, n}$ and the center of each edge segment is. assigned as the scattering center. Finally, shooting and bouncing rays (SBR) [5] is used to deal with multiple reflections. By this method, ray tubes are shot from a rectangular window on an incident wavefront, and a physical optics integration over a small region at the last bounce of each ray tube determines the amplitude and phase of a scattering center at 


$$
\bar{r}_{n}=\frac{1}{2}\left(\bar{r}_{1}+\bar{r}_{N}\right)-\frac{1}{2} \hat{\zeta} l_{N}
$$

where $\bar{r}_{1}$ is the first reflection point, $\bar{r}_{N}$ is the last reflection point, $d_{N}$ is the ray path length from $\bar{r}_{1}$ to $\bar{r}_{N}, \hat{\zeta}$ is the direction pointing from the target center to the receiver.

\section{Numerical Results}

To verify our approach, we consider two cases. The first one is the scattering from two orthogonal and separate rectangular plates. These two plates consist of 4 triangular facets

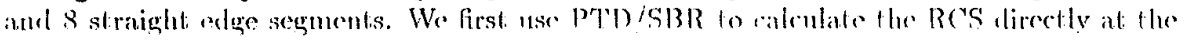
$\varphi=0$ plane. The aspect angle () varies trom - 50 to 90 degrees with the increment of one degree. The result is shown as Fig.2(a). We then use the method introduced in last section to obtain the scattering centers every 10 degrees at the same plane. For the scattering centers at certain $\theta$, we utilize Eq.(1) to reconstruct the RCS from $\theta-5$ degrees to $\theta+5$ degrees with the increment of one degree. The result is shown in Fig. 2(b). Both results agree very well. The second case is the scattering from an open rectangular cavity. This open cavity is composed of 5 rectangular plates (10 triangular facets and 12 straight edge segments). We again compute the RCS at the $\phi=0$ plane by the PTD/SBR method and by the point scatterer model. The PTD/SBR result is given in Fig.3(a). For the point scatterer model approach, we find the scattering centers every 6 degrees, and the RCS in each interval of 6 degrees is again evaluated by Eq.(1). The result is presented in Fig.3(b). The curves in Figs.3(a) and 3(b) are also in good agreement.

\section{Conclusions}

A new method for generating scattiering centers without resorting to the ISAR imaging has been proposed. Numerical results have shown that the RCS by our point scatterer model is consistent with that obtained through the direct PTD/SBR calculation. This new method is expected to be more efficient than those employing the ISAR imaging, because it is not necessary to collect scattering data of multiple frequencies and aspect angles to do the inverse Fourier transform.

\section{References}

[1] R. A. Ross and M. E. Bechtel, "Scattering-center theory and radar glint analysis," IEEE Trans. Aerospace and Electronic System, vol. AES-4, pp.756-762, Sept. 1968.

[2] M. S. McFarlin, "Generation of point scatterer models using RCS prediction codes," SCSC, pp.784-788, 1991.

(3] R. H. Delano, "A theory of target glint or angular scintillation in radar tracking;", Proc. IRE, vol.41, pp.1778-1784, Dec. 1953.

Y4] L. Peters, Jr., and F. C. Weimer, "Concerning the assumption of random distribution of scatterers as a model of an aircraft for tracking radar,"IRE Trans. Antennas and Propagation, vol. AP-9, pp.110-111, January, 1961. 
[5] H. Ling, R. Chou and S. W. Lee, "Shooling and bouncing rays: calculating the RCS of an arbitrarily shaped cavity," IEEE Trans. Antennas Propagatation, vol. AP-37, pp.194205, Feb. 1989.

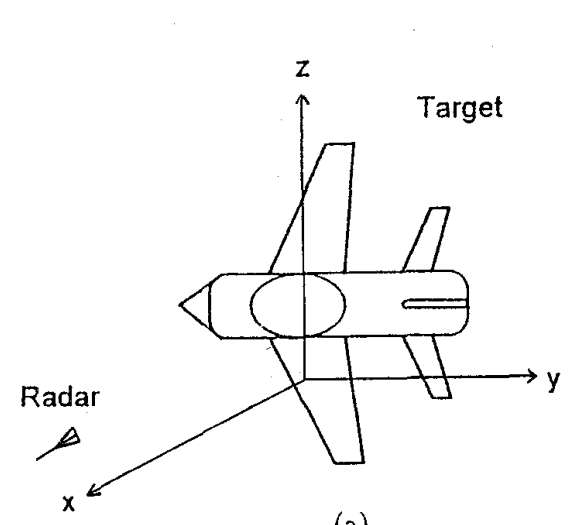

(a)

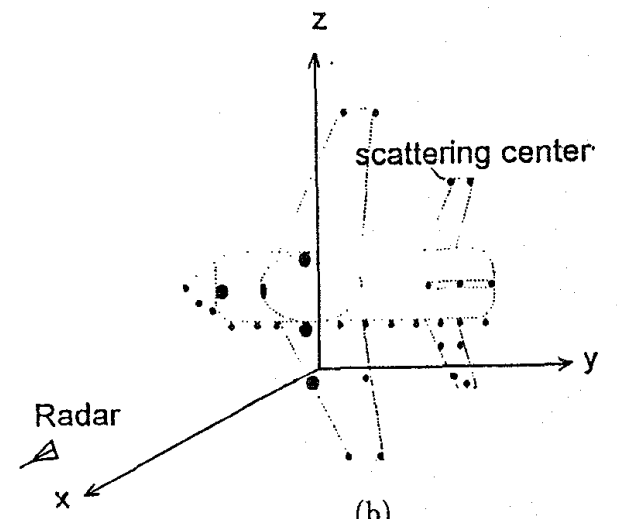

(b)

Fig. 1: (a). Target model.(b). Scattering centers of a target model.

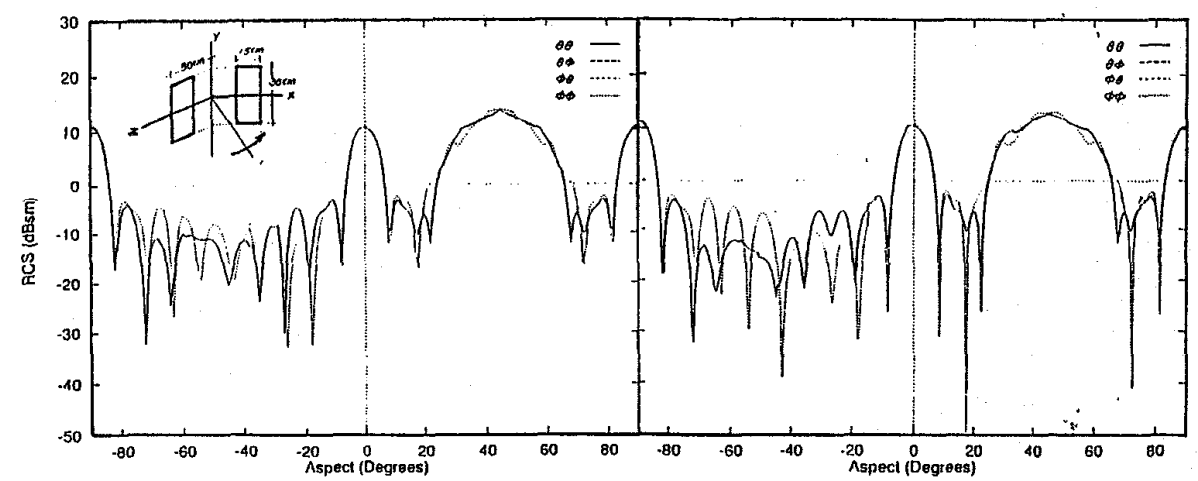

(a)

(b)

Fig. 2: (a).RCS of two orthogonal and separative plates by PTD/SBR method. (b).RCS of two orthogonal and separative plates by point scatterer model. 


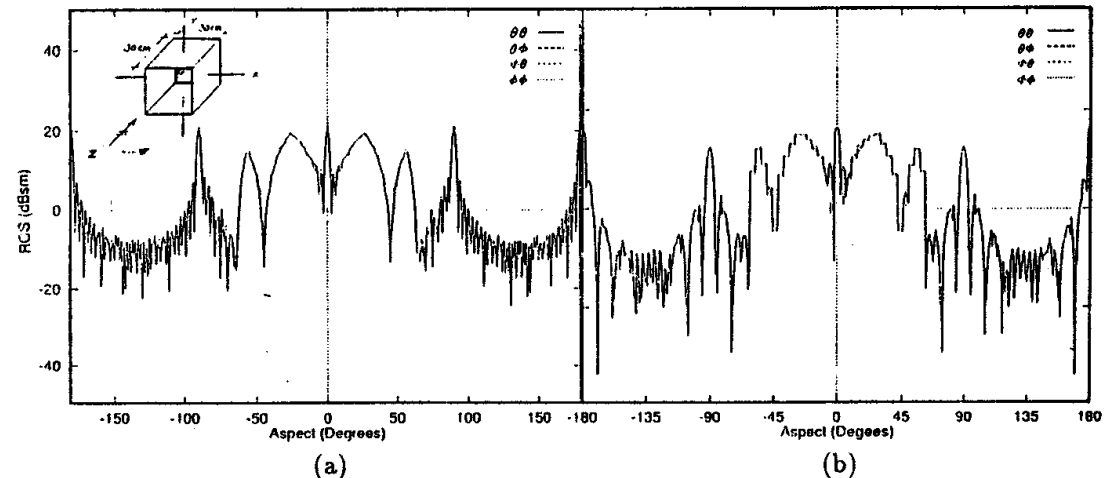

Fig. 3: (a).RCS of an open cavity by PTD/SBR method. (b).RCS of an open cavity by point scatterer model. 